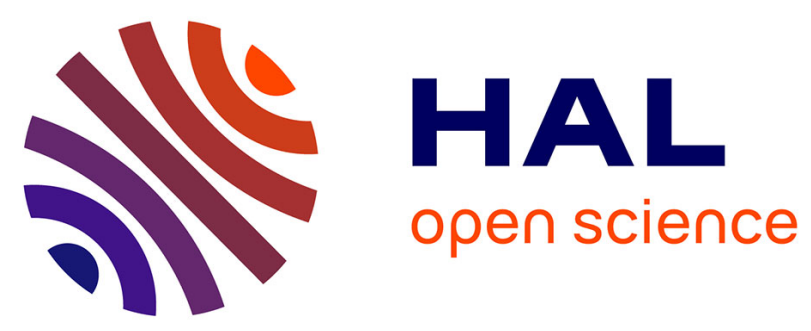

\title{
Risk factors for salivary gland cancers in France Results from a case-control study, the ICARE study
} Loredana Radoi, Christine Barul, Gwenn Menvielle, Matthieu Carton, Mireille Matrat, Marie Sanchez, Corinne Pilorget, Michel Velten, Isabelle Stucker, Danièle Luce

\section{To cite this version:}

Loredana Radoi, Christine Barul, Gwenn Menvielle, Matthieu Carton, Mireille Matrat, et al.. Risk factors for salivary gland cancers in France Results from a case-control study, the ICARE study. Oral Oncology, 2018, 80, pp. 56-63. 10.1016/j.oraloncology.2018.03.019 . hal-01795276

HAL Id: hal-01795276

https://hal-univ-rennes1.archives-ouvertes.fr/hal-01795276

Submitted on 9 Jun 2021

HAL is a multi-disciplinary open access archive for the deposit and dissemination of scientific research documents, whether they are published or not. The documents may come from teaching and research institutions in France or abroad, or from public or private research centers.
L'archive ouverte pluridisciplinaire HAL, est destinée au dépôt et à la diffusion de documents scientifiques de niveau recherche, publiés ou non, émanant des établissements d'enseignement et de recherche français ou étrangers, des laboratoires publics ou privés. 


\section{Risk factors for salivary gland cancers in France: Results from a case-control study, the ICARE study}

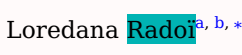

ICARE Study Group

aUniversity Paris Sud, Paris Saclay University, UVSQ, CESP, INSERM, Environmental Epidemiology of Cancer Team, Villejuif, France

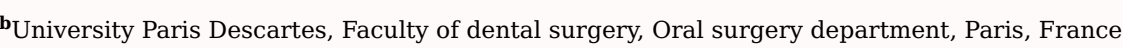

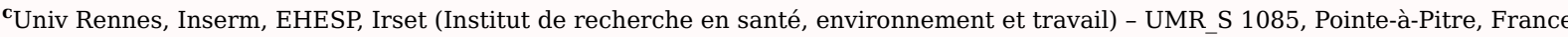

dSorbonne Universités, UPMC Univ Paris 06, INSERM, Institut Pierre Louis d'épidémiologie et de Santé Publique (IPLESP UMRS 1136), Paris, France

' Institut Curie, PSL Research University, DRCI, Biométrie, Saint-Cloud, France

fUniversité Paris Est Créteil, Faculté de médecine, Créteil, France

${ }^{\mathbf{g}}$ Centre Hospitalier Intercommunal, Service des Pathologies Professionnelles et de l’Environnement, Créteil, France

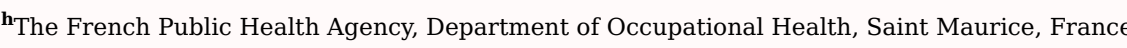

iUniversity Lyon, Claude Bernard Lyon1 University, Ifsttar, UMRESTTE, UMR T 9405, Lyon, France

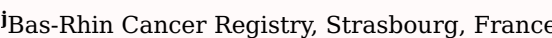

*Corresponding author at: Centre de Recherche en Épidémiologie et Santé des Populations, U1018, INSERM, Hôpital Paul Brousse, Bat 15 /16 16 av PV Couturier, 94807 Villejuif Cedex, France.

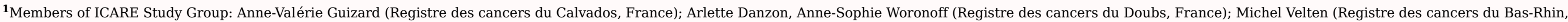

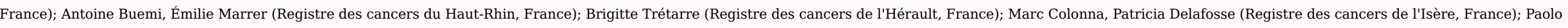

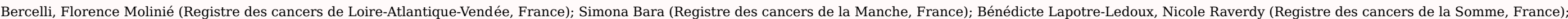

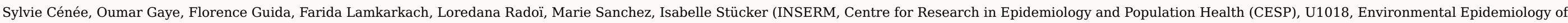

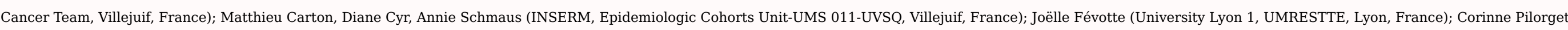




\section{Abstract}

\section{Objectives}

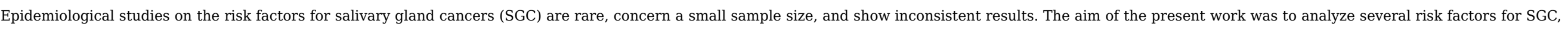
using the data from the ICARE study, a multicenter, population-based case-control study.

\section{Materials and methods}

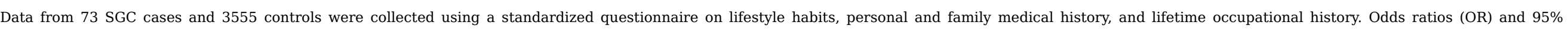
confidence intervals (CI) were estimated using unconditional logistic regressions.

\section{Results}

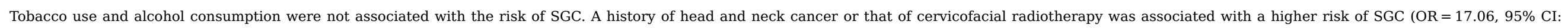

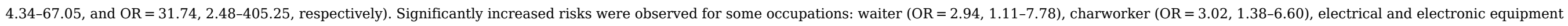

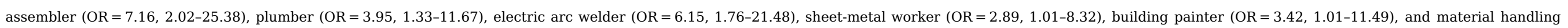
equipment operator $(\mathrm{OR}=5.05,1.71-14.84)$. Results for industries were consistent with those observed for occupations.

\section{Conclusion}

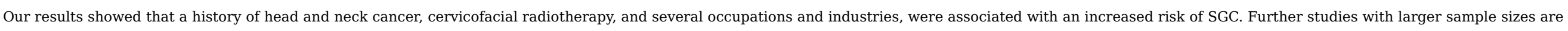
indicated to confirm our results.

Keywords: Salivary gland neoplasms; Head and neck cancers; Occupations; Industry; Epidemiologic studies; Risk factors; Life style

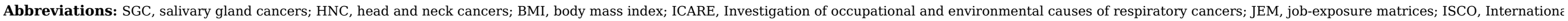
Standard Classification of Occupations; NAF, French Nomenclature of Activities; CEI, cumulative exposure index; OR, odds ratio; CI, confidence interval

\section{Introduction}

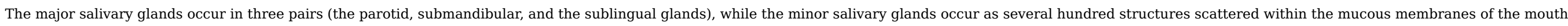

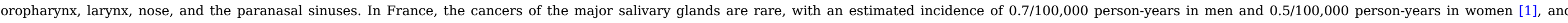
account for $1.9 \%$ and $8.6 \%$ of head and neck cancers (HNC) in men and in women, respectively [2]. The incidence rates of the cancers of the minor salivary glands have not been estimated.

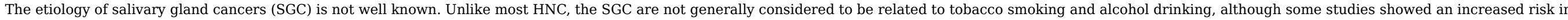

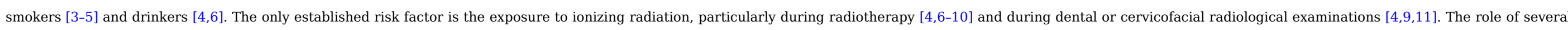

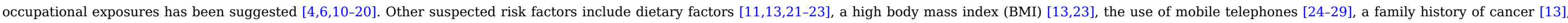
and certain viral infections [30-35].

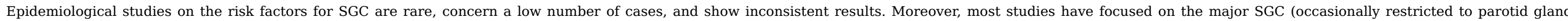

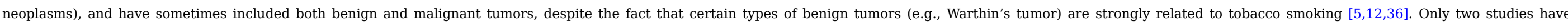
included both major and minor SGC [3,37].

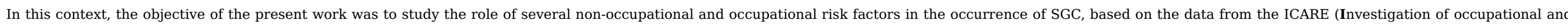
environmental CAuses of REspiratory cancers) study. 


\section{Materiel and methods}

\section{Study population}

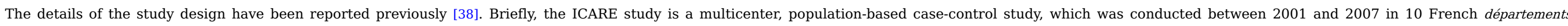

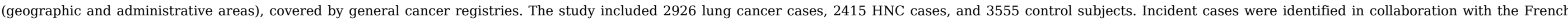

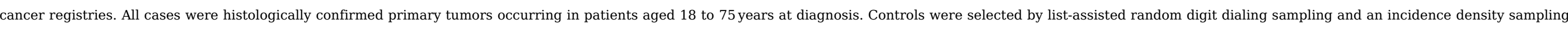

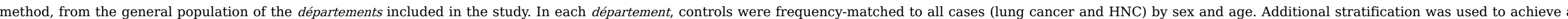
socioeconomic status distribution among the controls comparable to that of the general population of each département.

\section{Study sample}

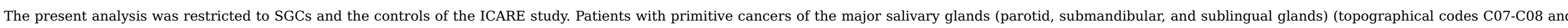

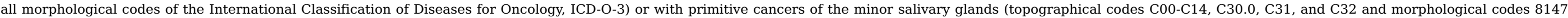

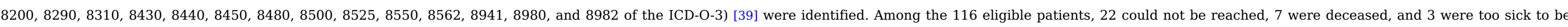

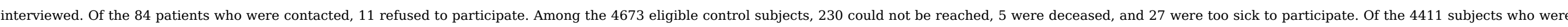

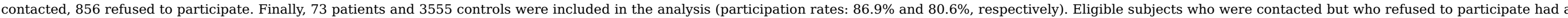
sex and age distribution similar to that of the included subjects:

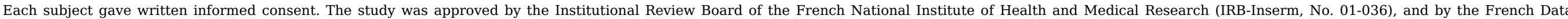
Protection Authority (CNIL No. 90120)

\section{Data collection}

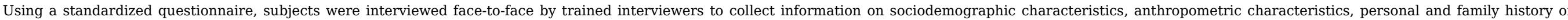
cancer, lifetime tobacco and alcohol consumption, and lifetime occupational history, with a detailed description of each job held for at least one month during the working life

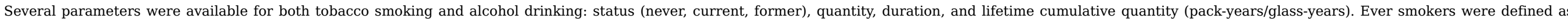

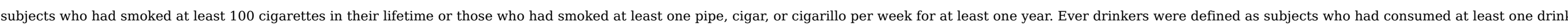

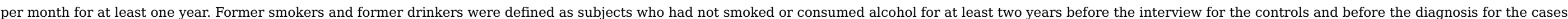
The quantity of tobacco smoked ( $\mathrm{g} /$ day) and alcohol consumed (standard glasses/day) were calculated by using the average lifetime daily consumption of all types of products consumed.

BMI was computed as weight $(\mathrm{kg})$ divided by height squared $\left(\mathrm{m}^{2}\right)$ and categorized into four classes: <18.5 (underweight), 18.5-24.9 (normal weight), 25.0-29.9 (overweight), and >30 (obesity).

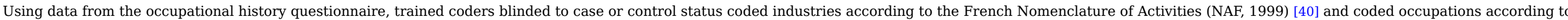
the International Standard Classification of Occupations (ISCO, 1968) [41].

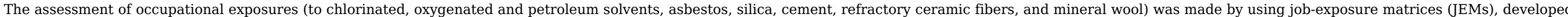
by the French Public Health Agency [42].

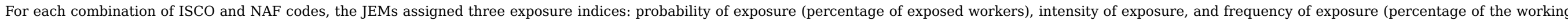

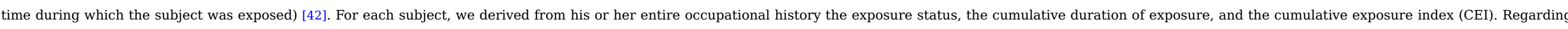

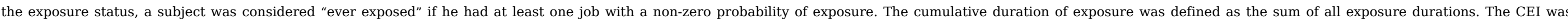
calculated as the sum of the values obtained by multiplying the weighted duration, probability, intensity, and frequency of exposure for each exposure period in the entire professional life.

\section{Statistical analysis}




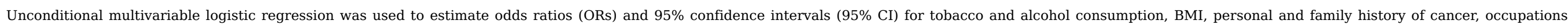

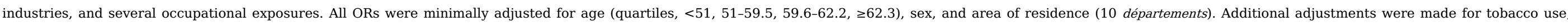

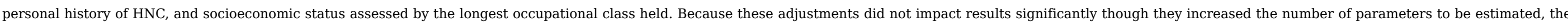
ORs were not adjusted for these variables.

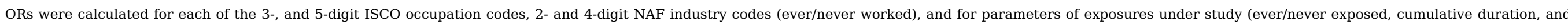

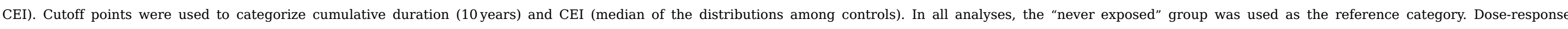

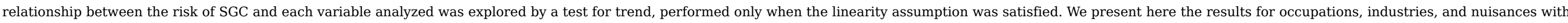
at least three exposed cases.

All tests were two-sided, and a p value $\leq 0.05$ was considered statistically significant. Statistical analyses were conducted using STATA software version 12.0 (StataCorp, TX, USA).

\section{Results}

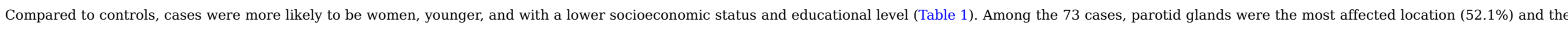
most frequent histological type was adenoid cystic carcinoma (21.9\%).

Table 1 Main characteristics of cases and controls and tumor location. The ICARE study.

\begin{tabular}{|c|c|c|c|}
\hline & Cases & Controls & $\mathrm{p}^{\mathrm{a}}$ \\
\hline & $\mathrm{n}=73(\%)$ & $\mathrm{n}=3555(\%)$ & \\
\hline Gender & & & 0.005 \\
\hline Male & $47(64.4)$ & $2780(78.2)$ & \\
\hline Female & $26(35.6)$ & $775(21.8)$ & \\
\hline Age (quartiles) & & & 0.09 \\
\hline Mean (SD) & $56.9(11.9)$ & $58.5(10.2)$ & \\
\hline$<51$ & $18(24.7)$ & $892(25.1)$ & \\
\hline 51-59.5 & $25(34.2)$ & $882(24.8)$ & \\
\hline $59.6-62.2$ & $10(13.7)$ & $906(25.5)$ & \\
\hline$\geq 62.3$ & $20(27.4)$ & $875(24.6)$ & \\
\hline Area of residence & & & 0.25 \\
\hline Calvados & $7(9.6)$ & $462(12.9)$ & \\
\hline Doubs, Territoire du Belfort & $1(1.4)$ & $143(4.0)$ & \\
\hline Herault & $12(16.4)$ & $450(12.7)$ & \\
\hline Isere & $4(5.5)$ & $501(14.1)$ & \\
\hline Loire Atlantique & $12(16.4)$ & $404(11.4)$ & \\
\hline Manche & $6(8.2)$ & $312(8.8)$ & \\
\hline Bas-Rhin & $9(12.3)$ & $469(13.2)$ & \\
\hline
\end{tabular}




\begin{tabular}{|c|c|c|c|}
\hline Haut-Rhin & $4(5.5)$ & $118(3.4)$ & \\
\hline Somme & $11(15.1)$ & $499(14.0)$ & \\
\hline Vendée & $7(9.6)$ & $197(5.5)$ & \\
\hline Socioeconomic status (based on the longest job held) & & & 0.10 \\
\hline Manager & 7 (9.6) & $618(17.4)$ & \\
\hline Farmer & $6(8.2)$ & $197(5.5)$ & \\
\hline Self-employed workers & $7(9.6)$ & $177(4.9)$ & \\
\hline Intermediate white-collar workers & $9(12.3)$ & 695 (19.5) & \\
\hline Office and sales employees & $16(21.9)$ & $672(18.9)$ & \\
\hline Blue-collar workers & $27(37)$ & $1178(33.1)$ & \\
\hline Educational level & & & 0.11 \\
\hline Primary or less & $18(24.7)$ & $763(21.5)$ & \\
\hline Vocational secondary & $28(38.4)$ & $1351(38.0)$ & \\
\hline General secondary & $7(9.6)$ & $400(11.3)$ & \\
\hline University & $12(16.4)$ & $901(25.3)$ & \\
\hline Others & $2(2.7)$ & $23(0.6)$ & \\
\hline \multicolumn{4}{|l|}{ Tumor location ${ }^{b}$} \\
\hline Parotid gland (C07.9) & $38(52.1)$ & & \\
\hline Submandibular gland (C08.0) & $9(12.3)$ & & \\
\hline Sublingual gland (C08.1) & $1(1.3)$ & & \\
\hline Major salivary gland, unspecified (C08.9) & $4(5.5)$ & & \\
\hline Minor salivary glands & $21(28.8)$ & & \\
\hline \multicolumn{4}{|l|}{ Histological type $^{c}$} \\
\hline Adenoid cystic carcinoma (8200) & $16(21.9)$ & & \\
\hline Mucoepidermoid carcinoma (8430) & $11(15.0)$ & & \\
\hline Squamous cell carcinoma (major salivary glands) $(8070,8071)$ & $10(13.7)$ & & \\
\hline Adenocarcinoma not otherwise specified (8140) & $9(12.3)$ & & \\
\hline Carcinoma ex pleomorphic adenoma (8941) & $4(5.5)$ & & \\
\hline Mucinous adenocarcinoma (8480) & $3(4.1)$ & & \\
\hline Epithelial myoepithelial carcinoma (8562) & $3(4.1)$ & & \\
\hline Others & $17(23.3)$ & & \\
\hline
\end{tabular}


$\mathrm{SD}=$ standard deviation.

a $p$ values are derived from the Pearson's chi-square test for categorical variables or Student's test for continuous variables.

$\mathbf{b}$ Topographical codes of the International Classification of Diseases for Oncology (ICD-O-3).

${ }^{\mathbf{c}}$ Morphological codes of the ICD-O-3.

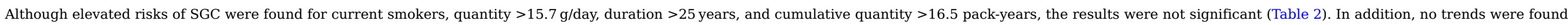

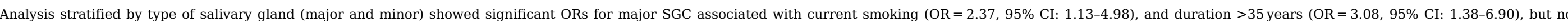
trend emerged. No association was found between minor SGC and tobacco consumption (not shown).

Table 2 Risk of salivary gland cancer associated with tobacco smoking. The ICARE study.

\begin{tabular}{|c|c|c|c|c|}
\hline & Cases & Controls & \multirow[t]{2}{*}{$\mathrm{OR}^{\mathrm{a}}(95 \% \mathrm{CI})$} & \multirow[t]{2}{*}{$\mathrm{p}$ trend } \\
\hline & $\mathrm{n}=73(\%)$ & $\mathrm{n}=3555(\%)$ & & \\
\hline \multicolumn{5}{|l|}{ Smoking status } \\
\hline Never smoker & 27 (36.9) & $1262(34.5)$ & Reference & \\
\hline Former smoker & $23(31.5)$ & $1461(41.1)$ & $0.98(0.53-1.79)$ & \\
\hline Current smoker & $23(31.5)$ & $820(23.1)$ & $1.63(0.88-3.01)$ & \\
\hline \multicolumn{5}{|c|}{ Quantity (g/day, quartiles) } \\
\hline Never smoker & $27(36.9)$ & $1262(34.5)$ & Reference & 0.66 \\
\hline \multicolumn{5}{|l|}{ Ever smoker } \\
\hline Mean (SD) & $16.9(12.5)$ & $17.8(14.2)$ & & \\
\hline $1-8.7$ & $12(16.4)$ & $563(15.8)$ & $1.20(0.59-2.44)$ & \\
\hline $8.7-15.7$ & $10(13.7)$ & $564(15.9)$ & $1.02(0.47-2.19)$ & \\
\hline $15.7-21.7$ & $13(17.8)$ & $561(15.7)$ & $1.48(0.72-3.03)$ & \\
\hline$>21.7$ & $11(15.1)$ & $563(15.8)$ & $1.31(0.61-2.83)$ & \\
\hline \multicolumn{5}{|c|}{ Duration (years, quartiles) } \\
\hline Never smoker & 27 (36.9) & $1262(34.5)$ & Reference & 0.49 \\
\hline \multicolumn{5}{|l|}{ Ever smoker } \\
\hline Mean (SD) & $27.7(13.8)$ & $25.1(13.3)$ & & \\
\hline $1-15$ & $12(16.4)$ & $611(17.2)$ & $1.12(0.54-2.30)$ & \\
\hline $16-25$ & $4(5.5)$ & $560(15.8)$ & $0.41(0.14-1.23)$ & \\
\hline $26-35$ & $15(20.5)$ & $554(15.6)$ & $1.59(0.80-3.16)$ & \\
\hline$>35$ & $15(20.5)$ & 555 (15.6) & $1.86(0.92-3.73)$ & \\
\hline
\end{tabular}




\section{Cumulative quantity (pack-years, quartiles)}

\begin{tabular}{|c|c|c|c|}
\hline Never smoker & 27 (36.9) & $1262(34.5)$ & Reference \\
\hline \multicolumn{4}{|l|}{ Ever smoker } \\
\hline Mean (SD) & $24.0(24.1)$ & $20.6(19.4)$ & \\
\hline $1-6.4$ & $13(17.8)$ & 561 (15.7) & $1.28(0.64-2.59$ \\
\hline $6.5-16.5$ & $6(8.2)$ & 571 (16.1) & $0.64(0.25-1.60$ \\
\hline $16.5-29$ & $12(16.4)$ & 562 (15.8) & $1.35(0.64-2.81)$ \\
\hline$>29$ & $14(19.2)$ & 554 (15.6) & $1.68(0.82-3.42)$ \\
\hline
\end{tabular}

$>29$

$14(19.2)$

$554(15.6)$

$1.68(0.82-3.42)$

$\mathrm{SD}=$ standard deviation; $\mathrm{OR}=$ odds ratio; $\mathrm{CI}=$ confidence interval.

${ }^{\text {a }}$ ORs were adjusted for age, sex, and area of residence.

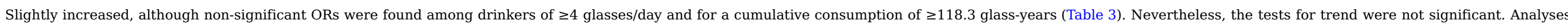
by type of salivary glands showed similar results for major SGC, but no associations with minor SGC were found (not shown).

Table 3 Risk of salivary gland cancer associated with alcohol consumption. The ICARE study.

\begin{tabular}{|c|c|c|c|c|}
\hline & Cases & Controls & \multirow[t]{2}{*}{ OR a $(95 \% \mathrm{CI})$} & \multirow[t]{2}{*}{$p$ trend } \\
\hline & $\mathrm{n}=73$ & $\mathrm{n}=3555$ & & \\
\hline \multicolumn{5}{|l|}{ Drinking status } \\
\hline Never drinker & 9 & 306 & Reference & \\
\hline Former drinker & 6 & 156 & $1.34(0.45-3.97)$ & \\
\hline Current drinker & 56 & 3071 & $0.68(0.31-1.46)$ & \\
\hline \multicolumn{5}{|c|}{ Quantity (glass/day, quartiles) } \\
\hline Never drinker & 9 & 306 & Reference & 0.16 \\
\hline \multicolumn{5}{|l|}{ Ever drinker } \\
\hline $0.03-0.44$ & 13 & 813 & $0.53(0.22-1.28)$ & \\
\hline $0.45-1.75$ & 12 & 804 & $0.54(0.21-1.38)$ & \\
\hline $1.76-3.95$ & 14 & 807 & $0.75(0.30-1.89)$ & \\
\hline$>3.95$ & 22 & 799 & $1.37(0.55-3.37)$ & \\
\hline \multicolumn{5}{|c|}{ Duration (years, quartiles) } \\
\hline Never drinker & 9 & 306 & Reference & 0.36 \\
\hline \multicolumn{5}{|l|}{ Ever drinker } \\
\hline $1-29$ & 17 & 864 & $0.66(0.26-1.66)$ & \\
\hline
\end{tabular}




\begin{tabular}{|c|c|c|c|c|}
\hline $30-38$ & 17 & 766 & $0.72(0.29-1.77)$ & \\
\hline $39-46$ & 16 & 797 & $0.92(0.37-2.29)$ & \\
\hline$\geq 47$ & 12 & 791 & $0.64(0.23-1.80)$ & \\
\hline \multicolumn{3}{|c|}{ Cumulative quantity (glass-years, quartiles) } & & \\
\hline Never drinker & 9 & 306 & Reference & 0.41 \\
\hline \multicolumn{5}{|l|}{ Ever drinker } \\
\hline $0.03-9.0$ & 14 & 805 & $0.57(0.24-1.38)$ & \\
\hline $9.1-47$ & 12 & 807 & $0.52(0.21-1.32)$ & \\
\hline $47.1-118.2$ & 16 & 803 & $0.83(0.33-2.06)$ & \\
\hline$\geq 118.3$ & 19 & 800 & $1.17(0.46-2.96)$ & \\
\hline
\end{tabular}

$\mathrm{SD}=$ standard deviation; $\mathrm{OR}=$ odds ratio; $\mathrm{CI}=$ confidence interval.

a ORs were adjusted for age, sex, and area of residence.

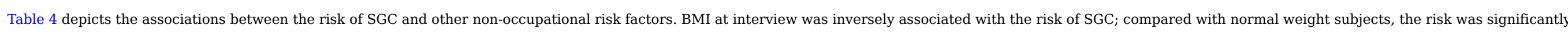

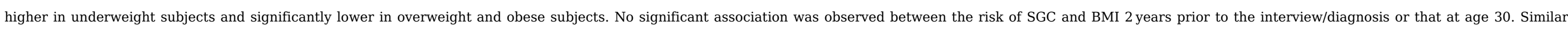
results were observed for major SGCs, but no associations were found for minor SGCs (not shown).

Table 4 Risk of salivary gland cancer associated with body mass index, history of cancer and of radiation therapy, and family history of head and neck cancer. The ICARE study.

\begin{tabular}{|c|c|c|c|c|}
\hline & Cases & Controls & \multirow{2}{*}{$\mathrm{OR}^{\mathrm{a}}(95 \% \mathrm{CI})$} & \multirow{2}{*}{$p$ trend } \\
\hline & $\mathrm{n}=73$ & $\mathrm{n}=3555$ & & \\
\hline \multicolumn{5}{|c|}{ BMI at interview/diagnosis } \\
\hline$<18.5$ & 4 & 37 & 3.55 (1.14-11.0) & \\
\hline $18.5-24.9$ & 31 & 1328 & Reference & 0.005 \\
\hline $25-29.9$ & 26 & 1441 & $0.77(0.44-1.32)$ & \\
\hline$\geq 30$ & 8 & 573 & $0.57(0.26-1.28)$ & \\
\hline \multicolumn{5}{|c|}{$B M I 2$ years prior the interview/diagnosis } \\
\hline$<18.5$ & 0 & 37 & - & \\
\hline $18.5-24.9$ & 26 & 1367 & Reference & 0.56 \\
\hline $25-29.9$ & 29 & 1367 & $1.18(0.68-2.05)$ & \\
\hline$\geq 30$ & 10 & 577 & $0.94(0.44-2.00)$ & \\
\hline \multicolumn{5}{|c|}{ BMI at age 30} \\
\hline$<18.5$ & 3 & 97 & $1.36(0.40-4.65)$ & \\
\hline
\end{tabular}




\begin{tabular}{|c|c|c|c|c|}
\hline $18.5-24.9$ & 41 & 2351 & Reference & 0.45 \\
\hline $25-29.9$ & 16 & 672 & $1.42(0.78-2.57)$ & \\
\hline$\geq 30$ & 2 & 123 & $0.99(0.23-4.20)$ & \\
\hline \multicolumn{5}{|l|}{ Previous history of cancer } \\
\hline No & 60 & 3090 & Reference & \\
\hline \multicolumn{5}{|l|}{ Yes } \\
\hline All cancer locations & 8 & 291 & $1.40(0.64-3.03)$ & \\
\hline Head and neck cancer & 3 & 13 & $17.06(4.34-67.05)$ & \\
\hline Prostate cancer & 1 & 78 & $0.74(0.10-5.64)$ & \\
\hline Hematological cancer & 1 & 16 & $2.85(0.35-23.08)$ & \\
\hline Other cancer locations & 3 & 184 & $0.73(0.22-2.41)$ & \\
\hline \multicolumn{5}{|c|}{ History of radiation therapy for cancer } \\
\hline No & 65 & 3265 & Reference & \\
\hline \multicolumn{5}{|l|}{ Yes } \\
\hline All cancer locations & 2 & 106 & $0.81(0.19-3.43)$ & \\
\hline Head and neck cancer & 1 & 2 & $31.74(2.48-405.25)$ & \\
\hline Hematological cancer & 1 & 8 & $5.10(0.56-46.20)$ & \\
\hline Other cancer locations & 0 & 96 & - & \\
\hline \multicolumn{5}{|c|}{ Family history of head and neck cancer } \\
\hline No & 56 & 2891 & Reference & \\
\hline$\geq 1$ first-degree relative & 4 & 166 & $1.18(0.42-3.34)$ & \\
\hline
\end{tabular}

$\mathrm{BMI}=$ body mass index; OR = odds ratio; $\mathrm{CI}=$ confidence interval.

${ }^{\text {a }}$ ORs were adjusted for age, sex, and area of residence.

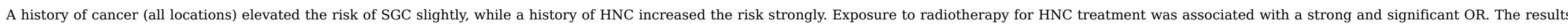
were consistent for major and minor salivary glands (not shown).

A family history of HNC in first-degree relatives was not significantly associated with a risk of SGC.

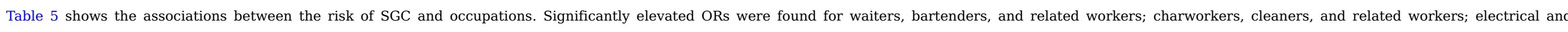

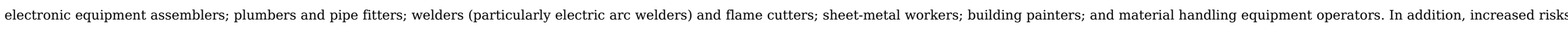

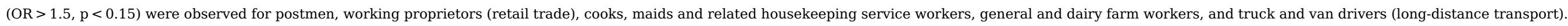

Table 5 Risk of salivary gland cancer associated with selected occupations according to the International Standard Classification of Occupations (ISCO, 1968). The ICARE study. 


\begin{tabular}{|c|c|c|}
\hline Occupation (ISCO codes) ${ }^{\mathrm{b}}$ & Exposed cases/controls & ORa $(95 \% \mathrm{CI})$ \\
\hline Secondary education teachers (1-32) & $4 / 227$ & $0.86(0.31-2.41)$ \\
\hline Primary education teachers $(1-33)$ & $3 / 131$ & $0.96(0.29-3.13)$ \\
\hline Teachers n.e.c (1-39) & $3 / 125$ & $1.41(0.43-4.60)$ \\
\hline Stenographers, typists and teletypists (3-21) & $3 / 140$ & $0.67(0.20-2.25)$ \\
\hline Bookkeepers and cashiers (3-31) & $4 / 228$ & $0.69(0.24-1.94)$ \\
\hline Mail distribution clerks (3-70) & $3 / 97$ & $1.45(0.44-4.78)$ \\
\hline Postman (3-70.30) & $3 / 45$ & $3.33(0.98-11.28)$ \\
\hline Working proprietors (wholesale and retail trade) (4-10) & $4 / 111$ & $1.81(0.64-5.14)$ \\
\hline Working proprietor (retail trade) $(4-10.30)$ & $4 / 97$ & $2.07(0.73-5.88)$ \\
\hline Salesmen, shop assistants and demonstrators (4-51) & $3 / 227$ & $0.47(0.14-1.55)$ \\
\hline Working proprietors (catering and lodging services) (5-10) & $3 / 59$ & $2.33(0.70-7.74)$ \\
\hline Cooks $(5-31)$ & $5 / 104$ & $2.04(0.79-5.27)$ \\
\hline Other cooks $(5-31.90)$ & $3 / 47$ & $2.36(0.69-8.01)$ \\
\hline Waiters, bartenders and related workers (5-32) & $6 / 96$ & $2.61(1.07-6.35)$ \\
\hline Waiter, general (5-32.10) & $5 / 71$ & $2.94(1.11-7.78)$ \\
\hline Maids and related housekeeping service workers n.e.c (5-40) & $8 / 144$ & $1.96(0.84-4.56)$ \\
\hline Housemaid (5-40.20) & $4 / 73$ & $1.81(0.60-5.47)$ \\
\hline Nursemaid (5-40.35) & $3 / 50$ & $1.75(0.50-6.10)$ \\
\hline Charworkers, cleaners and related workers (5-52) & $10 / 164$ & $2.47(1.16-5.22)$ \\
\hline Charworker (5-52.20) & $10 / 138$ & $3.02(1.38-6.60)$ \\
\hline General farmers (6-11) & $3 / 87$ & $1.65(0.49-5.56)$ \\
\hline Specialised farmers (6-12) & $3 / 169$ & $0.85(0.26-2.79)$ \\
\hline General farm workers (6-21) & $5 / 218$ & $1.11(0.43-2.89)$ \\
\hline Farm worker (general) (6-21.05) & $5 / 133$ & $1.97(0.74-5.18)$ \\
\hline Dairy farm workers (6-25) & $4 / 109$ & $2.21(0.74-6.58)$ \\
\hline Dairy farm worker (general) (6-25.10) & $4 / 106$ & $2.29(0.77-6.81)$ \\
\hline Blacksmiths, toolmakers and machine-tool operators n.e.c (8-39) & $3 / 77$ & $2.15(0.65-7.12)$ \\
\hline Electrical and electronic equipment assemblers (8-53) & $3 / 25$ & $7.16(2.02-25.38)$ \\
\hline Plumbers and pipe fitters (8-71) & $5 / 81$ & $3.51(1.33-9.29)$ \\
\hline Plumber (general) (8-71.05) & $4 / 54$ & 3.95 (1.33-11.67) \\
\hline Welders and flame cutters (8-72) & $6 / 77$ & 4.55 (1.86-11.12) \\
\hline
\end{tabular}


$\mathrm{OR}=$ odds ratio; $\mathrm{CI}=$ confidence interval; n.e.c $=$ not elsewhere classified.

a ORs were adjusted for age, sex, and area of residence.

b Only ISCO codes with at least 3 exposed cases are presented in this table.

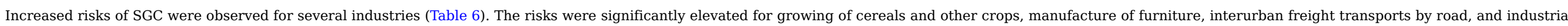

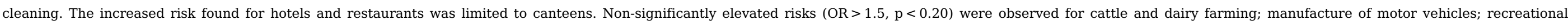
cultural, and sporting activities; other service activities; and household activities such as employers of domestic staff.

Table 6 Risk of salivary gland cancer associated with selected industries according to the French classification of activities (NAF, 1999). The ICARE study.

\begin{tabular}{|c|c|c|}
\hline Industry (NAF codes) ${ }^{b}$ & Exposed cases/controls & $\mathrm{OR}^{\mathrm{a}}(95 \% \mathrm{CI})$ \\
\hline Agriculture, hunting and related service activities (01) & $15 / 555$ & $1.44(0.77-2.56)$ \\
\hline Growing of cereals and other crops n.e.c. (01.1A) & $4 / 46$ & $5.15(1.71-15.44)$ \\
\hline Cattle farming, dairy farming (01.2A) & $3 / 90$ & $2.36(0.68-9.51)$ \\
\hline Growing of crops combined with farming of animals (01.3Z) & $6 / 299$ & $0.92(0.38-2.22)$ \\
\hline Manufacture of food products and beverages (15) & $4 / 320$ & $0.57(0.20-1.58)$ \\
\hline Manufacture of basic metals (27) & $3 / 78$ & $2.08(0.63-6.91))$ \\
\hline Manufacture of fabricated metal products, except machinery and equipment (28) & $3 / 241$ & $0.65(0.20-2.11)$ \\
\hline Manufacture of machinery and equipment n.e.c. (29) & $4 / 259$ & $0.87(0.31-2.45)$ \\
\hline Manufacture of motor vehicles, trailers and semi-trailers (34) & $5 / 201$ & $1.70(0.65-4.43)$ \\
\hline Manufacture of motor vehicles (34.1Z) & $4 / 120$ & $2.52(0.86-7.38)$ \\
\hline Manufacture of other transport equipment (35) & $5 / 125$ & $2.06(0.78-5.38)$ \\
\hline Manufacture of furniture; manufacturing n.e.c. (36) & $6 / 123$ & 2.77 (1.15-6.67) \\
\hline Construction (45) & $13 / 584$ & $1.35(0.71-2.57)$ \\
\hline
\end{tabular}


a ORs were adjusted for age, sex, and area of residence.

b Only NAF codes with at least 3 exposed cases are presented in this table.

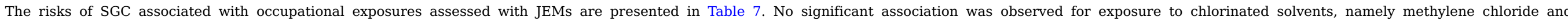

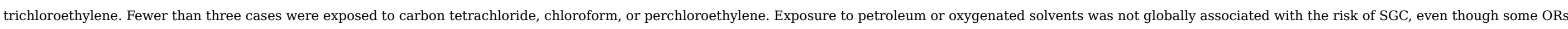
were slightly increased. However, no dose-response trend emerged for any solvent.

Table 7 Risk of salivary gland cancer associated with occupational exposures assessed through job-exposure matrices. The ICARE study.

\begin{tabular}{|c|c|c|c|c|c|c|}
\hline & \multirow{2}{*}{ Never exposed } & \multirow{2}{*}{ Ever exposed ${ }^{b}$} & \multicolumn{2}{|c|}{ Duration of exposure } & \multicolumn{2}{|c|}{ Cumulative Exposure Index } \\
\hline & & & $<10$ years & $\geq 10$ years & $<$ median & $\geq$ median \\
\hline
\end{tabular}




\begin{tabular}{|c|c|c|c|c|c|c|c|c|c|c|c|c|}
\hline & Cases/Controls & $\begin{array}{l}\mathrm{OR}^{\mathrm{a}}(95 \% \\
\mathrm{CI})\end{array}$ & Cases/Controls & $\begin{array}{l}\mathrm{OR}^{\mathrm{a}}(95 \% \\
\quad \mathrm{CI})\end{array}$ & Cases/Controls & $\mathrm{OR}^{\mathrm{a}}(95 \% \mathrm{CI})$ & Cases/Controls & $\begin{array}{l}\mathrm{OR}^{\mathrm{a}}(95 \% \\
\mathrm{CI})\end{array}$ & Cases/Controls & $\begin{array}{l}\mathrm{OR}^{\mathrm{a}}(95 \% \\
\quad \mathrm{CI})\end{array}$ & Cases/Controls & $\begin{array}{l}\mathrm{OR}^{\mathrm{a}}(95 \% \\
\quad \mathrm{CI})\end{array}$ \\
\hline \multicolumn{13}{|l|}{ Chlorinated solvents } \\
\hline Methylene chloride & $67 / 3253$ & $\begin{array}{l}1 \\
\text { (reference) }\end{array}$ & $5 / 276$ & $\begin{array}{l}0.99 \\
(0.39-2.51)\end{array}$ & $3 / 162$ & $\begin{array}{l}1.0 \\
(0.31-3.27)\end{array}$ & $2 / 114$ & $\begin{array}{l}0.96 \\
(0.23-4.04)\end{array}$ & $3 / 138$ & $\begin{array}{l}1.16 \\
(0.35-3.78)\end{array}$ & $2 / 138$ & $\begin{array}{l}0.81 \\
(0.19-3.39)\end{array}$ \\
\hline Trichloroethylene & $51 / 2449$ & $\begin{array}{l}1 \\
\text { (reference) }\end{array}$ & $22 / 1084$ & $\begin{array}{l}1.22 \\
(0.71-2.11)\end{array}$ & $9 / 544$ & $\begin{array}{l}0.97 \\
(0.46-2.03)\end{array}$ & $13 / 540$ & $\begin{array}{l}1.51 \\
(0.78-2.94)\end{array}$ & $12 / 542$ & $\begin{array}{l}1.27 \\
(0.65-2.47)\end{array}$ & $10 / 542$ & $\begin{array}{l}1.17 \\
(0.56-2.41)\end{array}$ \\
\hline At least one & $51 / 2397$ & $\begin{array}{l}1 \\
\text { (reference) }\end{array}$ & $22 / 1137$ & $\begin{array}{l}1.13 \\
(0.66-1.95)\end{array}$ & $8 / 557$ & $\begin{array}{l}0.82 \\
(0.38-1.79)\end{array}$ & $14 / 580$ & $\begin{array}{l}1.46 \\
(0.77-2.78)\end{array}$ & $9 / 568$ & $\begin{array}{l}0.91 \\
(0.43-1.91)\end{array}$ & $13 / 569$ & $\begin{array}{l}1.38 \\
(0.71-2.65)\end{array}$ \\
\hline \multicolumn{13}{|l|}{ Petroleum solvents } \\
\hline $\begin{array}{l}\text { Diesel, fuels and } \\
\text { kerosene }\end{array}$ & $50 / 2551$ & $\begin{array}{l}1 \\
\text { (reference) }\end{array}$ & $22 / 979$ & $\begin{array}{l}1.53 \\
(0.87-2.69)\end{array}$ & $12 / 507$ & $\begin{array}{l}1.55 \\
(0.78-3.08)\end{array}$ & $10 / 472$ & $\begin{array}{l}1.50 \\
(0.72-3.13)\end{array}$ & $12 / 488$ & $\begin{array}{l}1.67 \\
(0.84-3.30)\end{array}$ & $10 / 491$ & $\begin{array}{l}1.39 \\
(0.67-2.90)\end{array}$ \\
\hline Benzene & $60 / 2914$ & $\begin{array}{l}1 \\
\text { (reference) }\end{array}$ & $13 / 618$ & $\begin{array}{l}1.21 \\
(0.65-2.28)\end{array}$ & 9/394 & $\begin{array}{l}1.29 \\
(0.62-2.66)\end{array}$ & $4 / 224$ & $\begin{array}{l}1.07 \\
(0.37-3.05)\end{array}$ & 9/309 & $\begin{array}{l}1.63 \\
(0.79-3.36)\end{array}$ & $4 / 309$ & $\begin{array}{l}0.77 \\
(0.27-2.17)\end{array}$ \\
\hline White-spirits & $41 / 2009$ & $\begin{array}{l}1 \\
\text { (reference) }\end{array}$ & $32 / 1529$ & $\begin{array}{l}1.12 \\
(0.69-1.82)\end{array}$ & $14 / 650$ & $\begin{array}{l}1.08 \\
(0.58-2.02)\end{array}$ & $18 / 878$ & $\begin{array}{l}1.17 \\
(0.65-2.08)\end{array}$ & $15 / 764$ & $\begin{array}{l}0.98 \\
(0.53-1.79)\end{array}$ & $17 / 764$ & $\begin{array}{l}1.32 \\
(0.72-2.42)\end{array}$ \\
\hline $\begin{array}{l}\text { Other aliphatic mineral } \\
\text { spirits }\end{array}$ & $68 / 3239$ & $\begin{array}{l}1 \\
\text { (reference) }\end{array}$ & $4 / 294$ & $\begin{array}{l}0.69 \\
(0.25-1.93)\end{array}$ & $4 / 147$ & $\begin{array}{l}1.37 \\
(0.48-3.84)\end{array}$ & $0 / 147$ & - & $4 / 147$ & $\begin{array}{l}1.43 \\
(0.51-4.04)\end{array}$ & $0 / 147$ & - \\
\hline Gasoline & $60 / 2994$ & $\begin{array}{l}1 \\
\text { (reference) }\end{array}$ & $12 / 535$ & $\begin{array}{l}1.37 \\
(0.71-2.67)\end{array}$ & $8 / 308$ & $\begin{array}{l}1.55 \\
(0.71-3.38)\end{array}$ & $4 / 227$ & $\begin{array}{l}1.12 \\
(0.39-3.20)\end{array}$ & $7 / 267$ & $\begin{array}{l}1.60 \\
(0.70-3.66)\end{array}$ & $5 / 268$ & $\begin{array}{l}1.15 \\
(0.44-2.97)\end{array}$ \\
\hline At least one & $35 / 1783$ & $\begin{array}{l}1 \\
\text { (reference) }\end{array}$ & $38 / 1755$ & $\begin{array}{l}1.26 \\
(0.78-2.04)\end{array}$ & $12 / 655$ & $\begin{array}{l}0.98 \\
(0.50-1.92)\end{array}$ & 26/1099 & $\begin{array}{l}1.47 \\
(0.86-2.52)\end{array}$ & $17 / 876$ & $\begin{array}{l}1.04 \\
(0.57-1.88)\end{array}$ & $21 / 878$ & $\begin{array}{l}1.58 \\
(0.88-2.86)\end{array}$ \\
\hline \multicolumn{13}{|l|}{ Oxygenated solvents } \\
\hline Ethylene glycol & $68 / 3332$ & $\begin{array}{l}1 \\
\text { (reference) }\end{array}$ & $4 / 196$ & $\begin{array}{l}1.11 \\
(0.39-3.15)\end{array}$ & $2 / 104$ & $\begin{array}{l}1.00 \\
(0.23-4.23)\end{array}$ & $2 / 92$ & $\begin{array}{l}1.25 \\
(0.29-5.25)\end{array}$ & $2 / 99$ & $\begin{array}{l}1.03 \\
(0.24-4.36)\end{array}$ & $2 / 97$ & $\begin{array}{l}1.20 \\
(0.28-5.08)\end{array}$ \\
\hline Ketones and esters & $58 / 2815$ & $\begin{array}{l}1 \\
\text { (reference) }\end{array}$ & $15 / 717$ & $\begin{array}{l}1.15 \\
(0.63-2.07)\end{array}$ & $7 / 377$ & $\begin{array}{l}0.96 \\
(0.43-2.16)\end{array}$ & $8 / 340$ & $\begin{array}{l}1.38 \\
(0.64-2.96)\end{array}$ & $6 / 359$ & $\begin{array}{l}0.91 \\
(0.38-2.16)\end{array}$ & 9/358 & $\begin{array}{l}1.38 \\
(0.67-2.87)\end{array}$ \\
\hline Alcohols & $42 / 2234$ & $\begin{array}{l}1 \\
\text { (reference) }\end{array}$ & $31 / 1299$ & $\begin{array}{l}1.15 \\
(0.71-1.87)\end{array}$ & $13 / 612$ & $\begin{array}{l}0.99 \\
(0.52-1.87)\end{array}$ & $18 / 685$ & $\begin{array}{l}1.32 \\
(0.75-2.34)\end{array}$ & $11 / 641$ & $\begin{array}{l}0.84 \\
(0.43-1.66)\end{array}$ & $20 / 656$ & $\begin{array}{l}1.46 \\
(0.84-2.54)\end{array}$ \\
\hline At least one & $36 / 1735$ & $\begin{array}{l}1 \\
\text { (reference) }\end{array}$ & $37 / 1799$ & $\begin{array}{l}0.98 \\
(0.61-1.57)\end{array}$ & $15 / 724$ & $\begin{array}{l}0.94 \\
(0.51-1.75)\end{array}$ & $22 / 1074$ & $\begin{array}{l}1.01 \\
(0.59-1.74)\end{array}$ & $14 / 818$ & $\begin{array}{l}0.76 \\
(0.40-1.43)\end{array}$ & $23 / 900$ & $\begin{array}{l}1.19 \\
(0.69-2.03)\end{array}$ \\
\hline \multicolumn{13}{|l|}{ Other nuisances } \\
\hline Asbestos & $41 / 1786$ & $\begin{array}{l}1 \\
\text { (reference) }\end{array}$ & $32 / 1769$ & $\begin{array}{l}0.96 \\
(0.57-1.59)\end{array}$ & $12 / 567$ & $\begin{array}{l}1.06 \\
(0.54-2.08)\end{array}$ & $20 / 1202$ & $\begin{array}{l}0.90 \\
(0.50-1.62)\end{array}$ & $14 / 884$ & $\begin{array}{l}0.81 \\
(0.42-1.52)\end{array}$ & $18 / 885$ & $\begin{array}{l}1.15 \\
(0.62-2.13)\end{array}$ \\
\hline Mineral wool & $53 / 2619$ & $\begin{array}{l}1 \\
\text { (reference) }\end{array}$ & $19 / 914$ & $\begin{array}{l}0.81 \\
(0.45-1.44)\end{array}$ & $11 / 444$ & $\begin{array}{l}1.55 \\
(0.77-3.14)\end{array}$ & $8 / 470$ & $\begin{array}{l}1.03 \\
(0.46-2.28)\end{array}$ & $12 / 457$ & $\begin{array}{l}1.66 \\
(0.84-3.28)\end{array}$ & $7 / 457$ & $\begin{array}{l}0.92 \\
(0.40-2.13)\end{array}$ \\
\hline Silica & $57 / 2881$ & $\begin{array}{l}1 \\
\text { (reference) }\end{array}$ & $15 / 649$ & $\begin{array}{l}1.55 \\
(0.83-2.90)\end{array}$ & $7 / 327$ & $\begin{array}{l}1.39 \\
(0.61-3.20)\end{array}$ & $8 / 322$ & $\begin{array}{l}1.72 \\
(0.78-3.80)\end{array}$ & $7 / 327$ & $\begin{array}{l}1.43 \\
(0.62-3.28)\end{array}$ & $8 / 322$ & $\begin{array}{l}1.68 \\
(0.76-3.71)\end{array}$ \\
\hline
\end{tabular}




\begin{tabular}{|c|c|c|c|c|c|c|c|c|c|c|c|c|}
\hline Cement & $57 / 2990$ & $\begin{array}{l}1 \\
\text { (reference) }\end{array}$ & $15 / 539$ & $\begin{array}{l}1.86 \\
(0.99-3.48)\end{array}$ & $11 / 273$ & $\begin{array}{l}2.61 \\
(1.29-5.27)\end{array}$ & $4 / 266$ & $\begin{array}{l}1.04 \\
(0.36-2.98)\end{array}$ & $9 / 269$ & $\begin{array}{l}2.19 \\
(1.03-4.65)\end{array}$ & $6 / 270$ & $\begin{array}{l}1.52 \\
(0.62-3.69)\end{array}$ \\
\hline $\begin{array}{l}\text { Refractory ceramic } \\
\text { fibers }\end{array}$ & $67 / 3272$ & $\begin{array}{l}1 \\
\text { (reference) }\end{array}$ & $5 / 267$ & $\begin{array}{l}1.04 \\
(0.40-2.67)\end{array}$ & $0 / 115$ & - & $5 / 152$ & $\begin{array}{l}1.85 \\
(0.71-4.80)\end{array}$ & $1 / 134$ & $\begin{array}{l}0.41 \\
(0.06-3.05)\end{array}$ & $4 / 133$ & $\begin{array}{l}1.67 \\
(0.58-4.79)\end{array}$ \\
\hline
\end{tabular}

Cutoff point for Cumulative Exposure Index: median value of exposed controls.

a ORs were adjusted for age, sex, and area of residence.

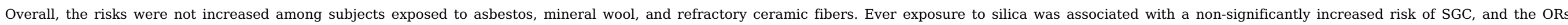

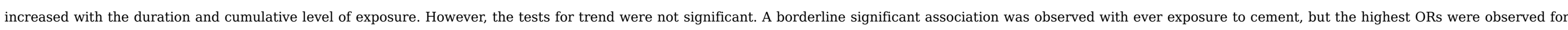

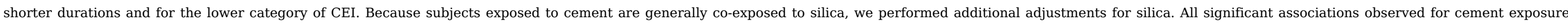

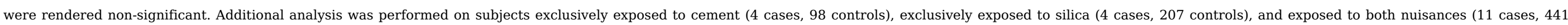
controls) (reference category: never exposed to cement, never exposed to silica). The corresponding ORs were 2.50 (95\% CI: 0.88-7.55), 1.46 (95\% CI: 0.50-4.23), and 1.83 (95\% CI: 0.88-3.78), respectively.

\section{Discussion}

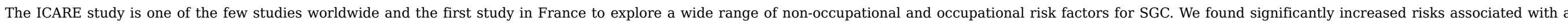
history of HNC and HNC-related radiotherapy, and elevated risks (non-significant) associated with tobacco use.

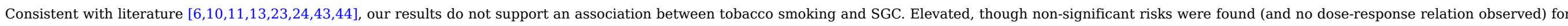
current smokers, for duration $>25$ years and for consumption >16.5 pack-years. Similarly, consistent with literature, no association between SGC and alcohol consumption was found [3,10-13,23,43,44].

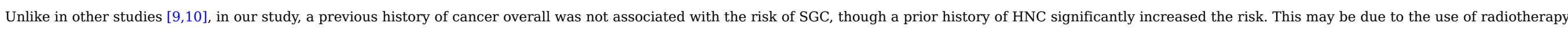

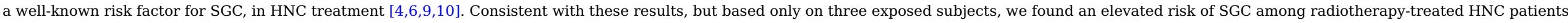

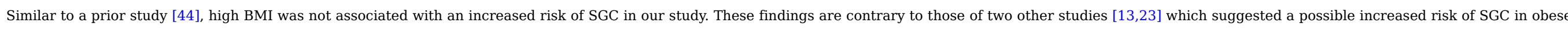

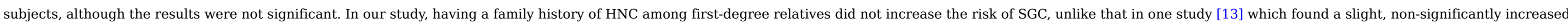
risk.

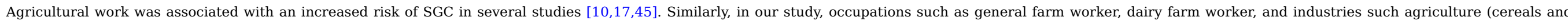
other crops) and cattle and dairy farming were associated with elevated risks of SGC, with ORs $>2$.

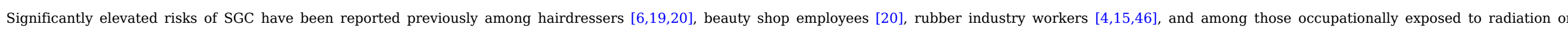

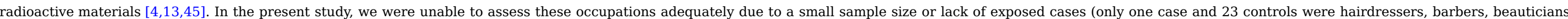
and related workers; there were no cases of rubber industry workers and radiation-exposed workers).

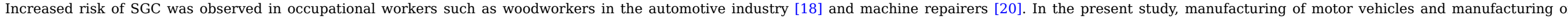

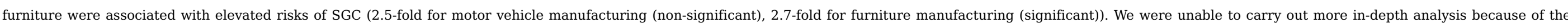
small number of subjects $(<3)$ in each 4 -digit industrial sector

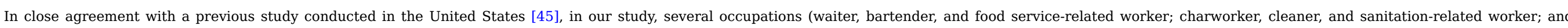

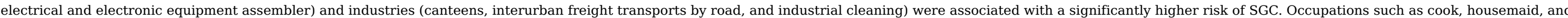
postman, and industries such as household services (as employers of domestic staff) were associated with an increased risk of SGC (ORs, approximately 2; non-significant).

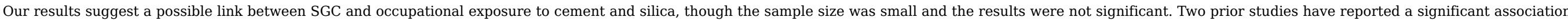
between the risk of SGC and exposure to cement [12] or silica [11] dust. Asbestos exposure was associated with elevated risks of SGC in two studies [12,14], but not in our study 


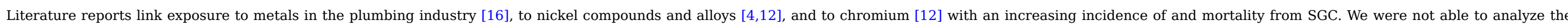

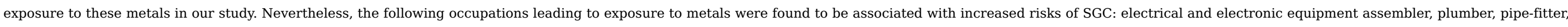

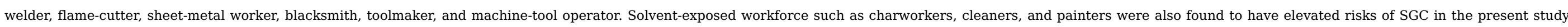

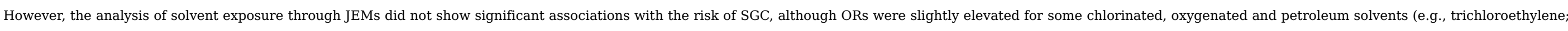

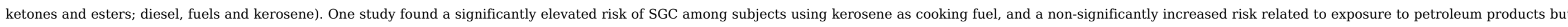

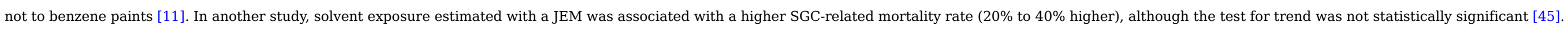

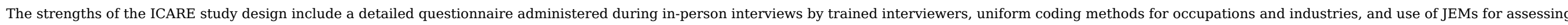
occupational exposure.

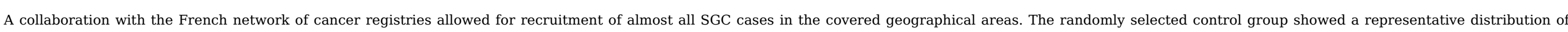

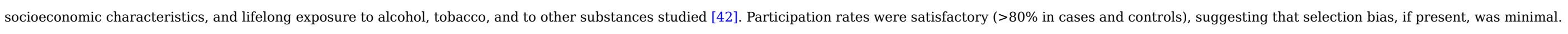

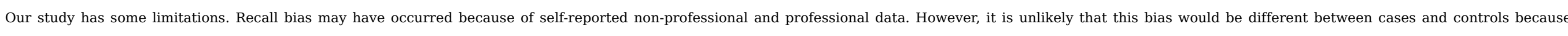

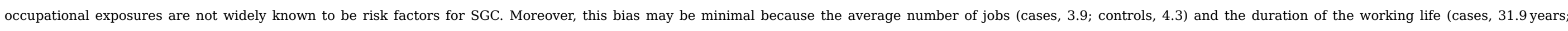
controls, 33.7 years) were comparable between groups.

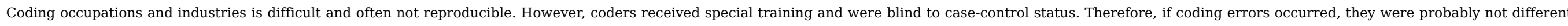

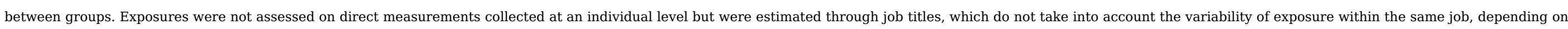

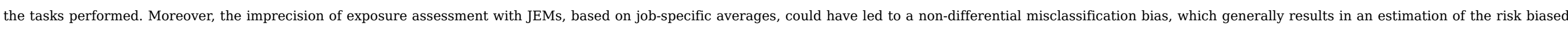

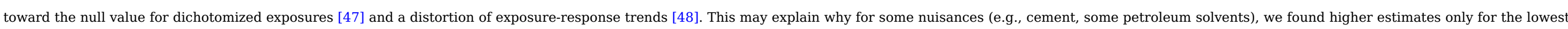
category of duration or CEI and no dose-response relation.

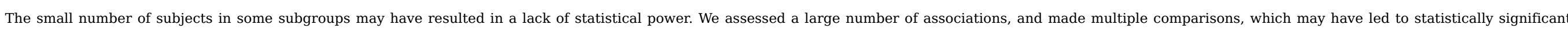

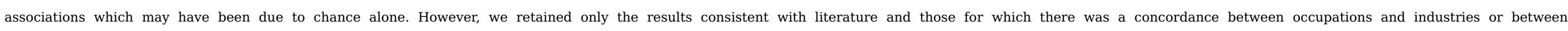
occupations/industries and possible exposures.

\section{Conclusion}

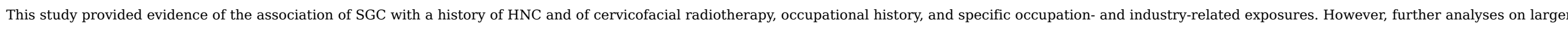
samples are required to confirm our results.

\section{Acknowledgements}

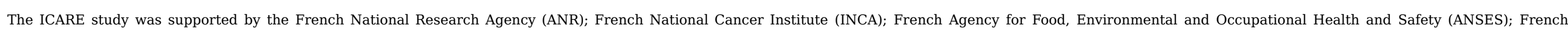

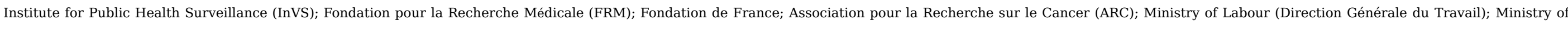
Health (Direction Générale de la Santé).

The authors thank all members of the MatGéné working group from Santé Publique France.

\section{Conflict of interest}

All authors declare that they have no conflict of interest.

\section{References}

[1] International Agency for Research on Cancer. Cancer Incidence in Five Continents. Volume X. IARC Scientific Publications n¹64, Lyon, France, 2014. 


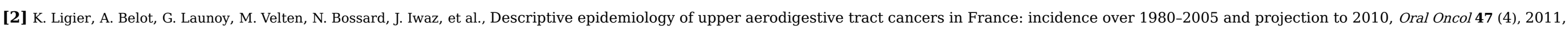
302-307.

[3] R.B. Hayes, E. Bravo-Otero, D.V. Kleinman, L.M. Brown, J.F. Fraumeni, Jr., L.C. Harty, et al., Tobacco and alcohol use and oral cancer in Puerto Rico, Cancer Causes Control 10 (1), 1999 , 27-33.

[4] P.L. Horn-Ross, B.M. Ljung and M. Morrow, Environmental factors and the risk of salivary gland cancer, Epidemiology. 8 (4), 1997, $414-419$.

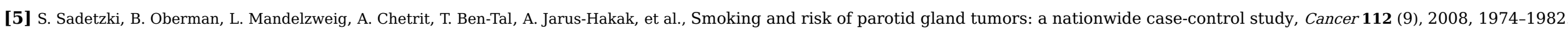

[6] M.R. Spitz, J.J. Fueger, H. Goepfert and G.R. Newell, Salivary gland cancer. A case-control investigation of risk factors, Arch Otolaryngol Head Neck Surg 116 (10), 1990, 1163-1166.

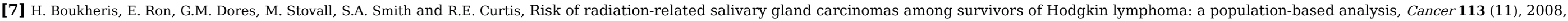
3153-3159.

[8] B. Modan, A. Chetrit, E. Alfandary, A. Tamir, A. Lusky, M. Wolf, et al., Increased risk of salivary gland tumors after low-dose irradiation, Laryngoscope 108 (7), 1998, 1095-1097.

[9] S. Preston-Martin, D.C. Thomas, S.C. White and D. Cohen, Prior exposure to medical and dental x-rays related to tumors of the parotid gland, J Natl Cancer Inst 80 (12), 1988, 943-949,

[10] M.R. Spitz, B.C. Tilley, J.G. Batsakis, J.M. Gibeau and G.R. Newell, Risk factors for major salivary gland carcinoma. A case-comparison study, Cancer 54 (9), 1984 , $1854-1859$.

[11] W. Zheng, X.O. Shu, B.T. Ji and Y.T. Gao, Diet and other risk factors for cancer of the salivary glands:a population-based case-control study, Int J Cancer $\mathbf{6 7}$ (2), $1996,194-198$.

[12] Dietz A, Barme B, Gewelke U, Sennewald E, Heller WD, Maier H. The epidemiology of parotid tumors. A case control study. Hno. 1993;41(2):83-90.

[13] J. Forrest, P. Campbell, N. Kreiger and M. Sloan, Salivary gland cancer: an exploratory analysis of dietary factors, Nutr Cancer 60 (4), $2008,469-473$.

[14] S. Graham, M. Blanchet and T. Rohrer, Cancer in asbestos-mining and other areas of Quebec, J Natl Cancer Inst 59 (4), 1977, $1139-1145$.

[15] T.F. Mancuso and M.J. Brennan, Epidemiological considerations of cancer of the gallbladder, bile ducts and salivary glands in the rubber industry, J Occup Med 12 (9), 1970, 333-341.

[16] S. Milham, Jr., Cancer mortality pattern associated with exposure to metals, Ann NY Acad Sci 271, 1976, 243-249.

[17] J.H. Olsen, L. Dragsted and H. Autrup, Cancer risk and occupational exposure to aflatoxins in Denmark, BrJ Cancer 58 (3), 1988, 392-396.

[18] G.M. Swanson and S.H. Belle, Cancer morbidity among woodworkers in the U.S. automotive industry, J Occup Med 24 (4), 1982, 315-319.

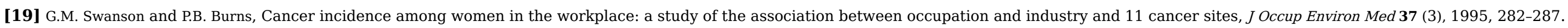

[20] G.M. Swanson and P.B. Burns, Cancers of the salivary gland: workplace risks among women and men, Ann Epidemiol 7 (6), 1997, 369-374.

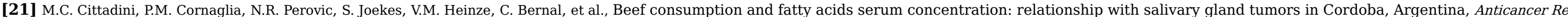
34 (10), 2014, 5579-5584

[22] P.L. Horn-Ross, M. Morrow and B.M. Ljung, Diet and the risk of salivary gland cancer, Am J Epidemiol 146 (2), 1997, 171-176.

[23] S.Y. Pan, M. de Groh and H. Morrison, A case-control study of risk factors for salivary gland cancer in Canada, J Cancer Epidemiol 2017, 2017, 4909214.

[24] A. Auvinen, M. Hietanen, R. Luukkonen and R.S. Koskela, Brain tumors and salivary gland cancers among cellular telephone users, Epidemiology 13 (3), 2002, 356-359.

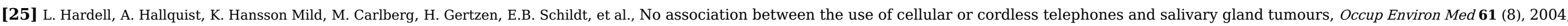
675-679.

[26] S. Lonn, A. Ahlbom, H.C. Christensen, C. Johansen, J. Schuz, S. Edstrom, et al., Mobile phone use and risk of parotid gland tumor, Am J Epidemiol 164 (7), 2006, 637-643.

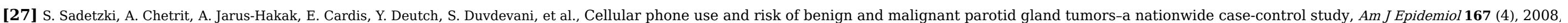


457-467.

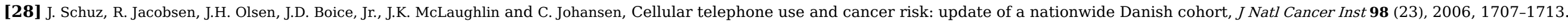

[29] F. Soderqvist, M. Carlberg and L. Hardell, Use of wireless phones and the risk of salivary gland tumours: a case-control study, Eur J Cancer Prev 21 (6), 2012 , 576-579.

[30] O. Gallo, Increasing evidence that Epstein-Barr virus may be involved in the pathogenesis of undifferentiated carcinoma of the salivary glands, Hum Pathol 27 (12), $1996,1381$.

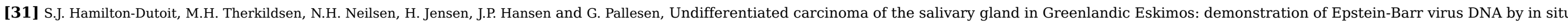
nucleic acid hybridization, Hum Pathol 22 (8), 1991, 811-815.

[32] A.P. Lanier and S.R. Alberts, Cancers of the buccal cavity and pharynx in Circumpolar Inuit, Acta Oncol 35 (5), 1996, 545-552.

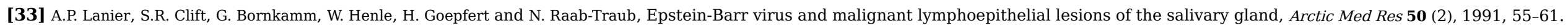

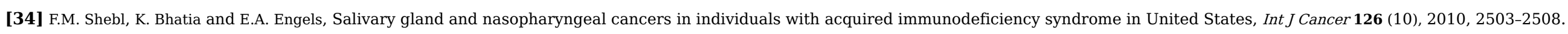

[35] D. Vageli, G. Sourvinos, M. Ioannou, G.K. Koukoulis and D.A. Spandidos, High-risk human papillomavirus (HPV) in parotid lesions, Int J Biol Markers 22 (4), 2007 , $239-244$.

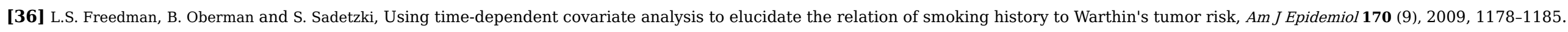

[37] M.R. Spitz, J.G. Sider and G.R. Newell, Salivary gland cancer and risk of subsequent skin cancer, Head Neck 12 (3), 1990, $254-256$.

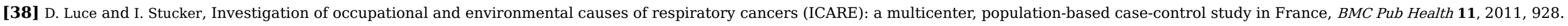

[39] World Health Organization, Int Classif Dis Oncol, third ed, 2000, World Health Organization; Geneva.

[40] Institut National de la Statistique et des Etudes Economiques. Nomenclature d'activités et de produits française, NAF. INSEE, Paris, France, 1999.

[41] International Labour Office, Int Stand Classif Occup, 1968, International Labour Office; Geneva.

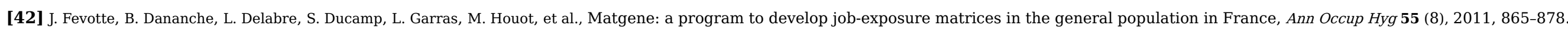

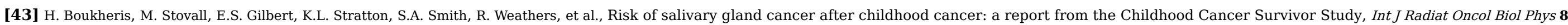
(3), 2013, 776-783.

[44] J.E. Muscat and E.L. Wynder, A case/control study of risk factors for major salivary gland cancer, Otolaryngol Head Neck Surg 118 (2), 1998, 195-198.

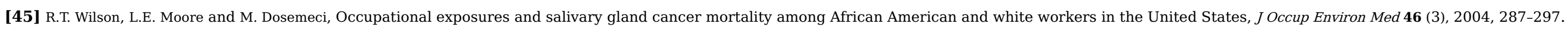

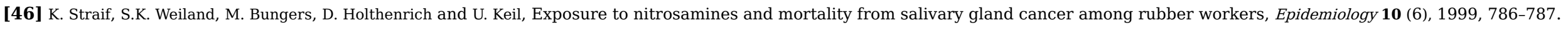

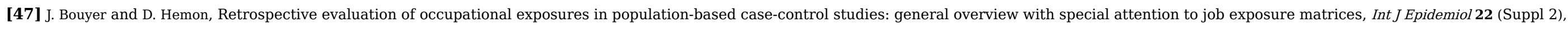
1993, S57-S64.

[48] M. Dosemeci, S. Wacholder and J.H. Lubin, Does nondifferential misclassification of exposure always bias a true effect toward the null value?, AmJ Epidemiol 132 (4), 1990 , 746-748.

\section{Highlights}

- Tobacco and alcohol consumption did not increase the risk of salivary gland cancer.

- History of head and neck cancer and cervicofacial radiotherapy increased the risk. 
